\title{
PET in uterine malignancies
}

\author{
Valeria Pirro ${ }^{1}$, Andrea Skanjeti ${ }^{1}$, Ettore Pelosi ${ }^{2 *}$ \\ ${ }^{1}$ Nuclear Medicine Unit, San Giovanni Battista Hospital, University of Turin, Turin, Italy \\ ${ }^{2}$ PET Centre, IRMET S.p.A., Turin, Italy; ${ }^{*}$ Corresponding Author: e.pelosi@irmet.com
}

Received 7 March 2010; revised 2 April 2010; accepted 5 May 2010.

\begin{abstract}
Positron Emission Tomography (PET) or integrated PET/Computed Tomography (PET/CT) with ${ }^{18}$ F-Fluoro-Deoxy-Glucose $\left({ }^{18}\right.$ F-FDG $)$ is a functional imaging modality, useful in the characterization of undetermined morphological findings, and in the staging/re-staging of a large number of malignancies. Although its use in uterine malignancies has been poorly investigated, in recent years the employment of this technique has constantly increased. In this review, we evaluate the role of PET (ICT) with ${ }^{18} \mathrm{~F}$ FDG in uterine malignancies (cervical and endometrial cancers as well as uterine sarcomas), underlying its advantages and discussing its limitations. Metabolic and anatomic information given by PET/CT with ${ }^{18}$ F-FDG could be useful in the evaluation of local and distant disease involvement at the staging, in the detection of disease recurrence, and in the evaluation of the response after chemotherapy and/or radio-therapy.
\end{abstract}

Keywords: ${ }^{18}$ F-FDG PET/CT; Uterine Malignancies; Cervical Cancer; Endometrial Cancer;

Uterine Sarcomas

\section{INTRODUCTION}

Positron Emission Tomography (PET) or integrated PET/Computed Tomography (PET/CT) with ${ }^{18}$ F-FluoroDeoxy-Glucose $\left({ }^{18} \mathrm{~F}-\mathrm{FDG}\right)$ study is a functional, non invasive whole body examination, that allows to metabolically characterize undetermined morphological findings, stage/re-stage disease, evaluate treatment response and monitor the therapy in a large number of malignancies (lymphomas, lung, breast, colon-rectal cancer, etc). In the genital tract of women the use of PET is controversial and it is limited by the urinary excretion of the

${ }^{18} \mathrm{~F}$-FDG, that interfere with the evaluation of uterus and vagina, and by the numerous false positive findings related to the presence of physiological tracer uptakes in the bowel, ovaries, and in the uterus itself. However, the clinical introduction of integrated PET/CT tomography, allowing the co-registration and the superimposition of anatomical and functional images and thus the exact localization of all the ${ }^{18}$ F-FDG uptakes, improved PET diagnostic accuracy [1].

The aim of the present review is to discuss the role of ${ }^{18} \mathrm{~F}$-FDG-PET or PET/CT (PET/(CT)) exam in the uterine cervical cancer, in the endometrial adenocarcinoma and in the uterine sarcoma.

\section{LITERATURE SEARCH}

For this review, in the matter of the role of ${ }^{18} \mathrm{~F}$-FDG-PET (/CT) in the above mentioned gynaecological malignancies, a MEDLINE search has been performed in order to find relevant articles. For all the evaluated malignancies we included only primary studies and meta-analysis published in the English language in the last five years. We did not include case reports, and abstracts. For uterine cervical cancer we used as keywords: uterine cancer, cervical cancer, and uterine cervix carcinoma; while for endometrial cancer we used: uterine corpus carcinoma or neoplasm, and endometrial cancer. Finally, in the case of uterine sarcomas, we used as keywords uterine sarcoma, uterine carcinosarcoma, and uterine leyomiosarcoma. In all of the above mentioned cases, each keyword was always associated with Positron Emission Tomography, PET/CT, and ${ }^{18}$ F-FDG-PET/CT. Furthermore, to complete the search we look for in the bibliography of the founded studies and considered the most recent and interesting works.

\section{CERVICAL CANCER}

Cervical cancer is the third most common neoplasm in women. Recent data report an incidence rate of about 42,000 new cases/year in the United States and in the European Union [2,3] and 150,000 deaths/year world- 
wide [4]. In the last decades, the introduction of the $\mathrm{Pa}$ panicolau screening test (Pap-test) has allowed an increase in the detection rate of pre-invasive lesions; in the same time the mortality due to the invasive cervical cancer has not substantially decreased $[5,6]$.

To stage cervical cancer the International Federation of Gynaecologists and Obstetrics (FIGO) staging system is currently used $[7,8]$. In patients with small localized carcinomas (stage IA and IB1) radical hysterectomy or radiotherapy alone are equally recommended [9]. For large lesions and/or locally advanced cancers (stage IB2IVA), chemo-radiotherapy is the treatment of choice [10,11]. Tumour size, parametrial tissue involvement, pelvic and/or para-aortic lymph node spread and deep invasion of nearby organs are the most important prognostic parameters at the diagnosis [12]. Patients with these unfavourable prognostic factors are at high risk of developing disease recurrence with an estimated recurrence rate ranging between $23 \%$ and $35 \%$ [13,14].

Even if the assessment of local and distant disease extension is a crucial point both in the pre- and post-treatment phases, however a standardized protocol to stage/ re-stage these patients has not been established [15]. In particular, no imaging modalities are routinely used in this work up, which is based on physical examination, Pap-test, serum markers assay and surgical evaluation. Currently, the use of ${ }^{18} \mathrm{~F}$-FDG-PET/CT in the management of patients with cervical cancer has been investigated in different settings.

\subsection{Staging}

The assessment of the primary lesion is actually based on the clinical examination and on morphological imaging modalities, in particular MRI. One of the crucial data is the presence of uterine parametrial invasion. In this field, despite the presence of co-registered CT images, the diagnostic performance of ${ }^{18} \mathrm{~F}$-FDG-PET/CT is worse than MRI, due to the lack of a good spatial resolution. In the local staging of 32 primary tumours, Park et al. highlighted a higher number of false negative results at PET scan than at MRI (3 and 1 case, respectively) [9]. Probably, in stage IA or IB the amount of disease is under or at the limit of the PET system resolution and its detection can be elusive. Moreover, the interference of urinary activity, that of physiological processes (such as hormone-dependent changes in the ovaries and endometrium during the phases of menstrual cycle) and some benign pathologies (such as corpus luteum cysts, endometriosis, inflammations, menstruations, etc.) can interfere with the optimal evaluation of the primary lesion, leading to difficulties in exam interpretation $[12,16]$. Some of these PET limitations can be reduced with practical expedients: for example, the urinary interference can be avoided by emptying the bladder just before the start of the exam, or by the hydration and administration of diuretics, or by the continuous bladder irrigation to dilute and remove the radioactive urine. On the basis of these limitations, it is clear that, despite the on-going technical improvements, PET is still unsatisfactory in the evaluation of the primary lesion and particularly in the identification of the deep uterine tissue involvement.

On the other hand, an application of PET exam in these cases is actually under debate, i.e. the prognostic value of the primary lesion ${ }^{18} \mathrm{~F}$-FDG uptake. The tumour ${ }^{18}$ F-FDG uptake, generally measured by the maximum Standardized Uptake Value (SUVmax), seems to be strictly related to the behaviour and to the aggressiveness of the tumour itself as in other malignancies (head and neck, lung and oesophageal cancers). In uterine cancer different authors reported an independent correlation between the cancer SUVmax and: the lymph node status, the disease response to chemo/radio therapy, the frequency of pelvic recurrence, the disease free and the overall survival [12,17-20]. In a study on 240 patients, Kidd et al. [21] confirmed the correlation between the SUVmax at the staging and the presence of lymph node metastases. Moreover, Lee and colleagues [22] observed a good correlation between SUVmax and DFS in early stages of cervical cancer. From these experiences, it seems that SUVmax could be a useful prognostic tool in this cancer too.

In the pre-treatment disease staging, the identification of nodal (loco-regional and para-aortic) and distant metastasis are crucial points, which present prognostic and therapeutic significance. In fact, in locally advanced cervical cancer the 5 year survival rate is $57 \%$, $34 \%$ or $12 \%$, in node negative cases, pelvic nodes metastasis or para-aortic nodes metastasis, respectively [23]. In a recent meta-analysis, the most accurate method to study lymph node involvement resulted to be the sentinel node biopsy; however, this is a (minimal) invasive procedure, that often requires the administration of anaesthetic drugs, and that could lead to some complications. Among the imaging tests, the authors of this meta-analysis affirm that PET/CT presents better accuracy than contrast enhancement (CE) CT and MRI and that it could be used to guide laparoscopic staging procedures [24]. In fact, the sensitivity of CECT and/or MRI in identifying nodal metastasis is very low. A Gynaecological Oncology Group (GOG) study reported a sensitivity of 34\% in the detection of para-aortic lymph nodes by CECT [25]. Furthermore, in patients with gynaecological cancer and CECT negative for lymph node metastasis, PET/CT showed sensitivity and specificity of $50 \%$ and $83.3 \%$ respectively [25]. The good accuracy of PET and PET/CT scans in detecting lymph nodes has been established by 
the meta-analysis of Havrilesky et al., that assessed a pooled sensitivity and specificity rate of $84 \%$ and $95 \%$ for para-aortic lymph nodes and $79 \%$ and $99 \%$ for pelvic lymph nodes [26]. More recently, other authors confirmed these results for PET [27-32]. Furthermore, it was evidenced an increase in the accuracy for combined PET/CT [7,25,33-36]. Finally, Yen et al, indicate that an SUVmax of para-aortic lymph nodes greater than 3.3 is a strong negative prognostic factor in patients with locally advanced disease in respect to recurrence and survival rate [36]. On the other hand as reported by several authors, the limit of PET in this field, is represented by the significant number of false negative lymph nodes. This pitfall is related to the limited spatial resolution of the tomograph [31,37-39]. Kitajima et al. [40] attempted to improve the accuracy of the exam performing a PET/ CECT scan. They found a per patient based sensitivity, specificity, positive predictive value, negative predictive value and accuracy of 50\%, 90.9\%, 66.7\%, 83.3\% and $80 \%$, respectively, and per lymph nodes based sensitivity specificity, positive predictive value, negative predictive value and accuracy of 51.1\%, 99.8\%, 85.2\%, 98.9\% and $98.7 \%$, respectively. On the other hand, Kim et al. [23] advise the use of fused MRI/PET to increase the detection of lymph node metastasis. In conclusion, due to the low sensitivity, the use of PET/CT study to stage lymph nodes should be taken into account only for patients presenting important co-morbidities and/or contraindications to the surgical approach.

\subsection{Radiotreatment Planning}

Radiation treatment is indicated in a large part of these patients. The accurate definition of the treatment planning is mandatory in order to adequately radiate the tumour and to spare near critical organs. As in other malignancies, the target volume of radiation beam is currently based on morphological examinations (such as CECT and MRI), that allow high spatial resolution images with accurate anatomical definition [41]. However, this staging modality presents some limitations [42] and the adjunct of the metabolic study significantly improve the identification of the target volumes, allowing the identification of the viable part of the tumour, and improving the staging $[43,44]$. When PET is used, the Planning Target Volume in modified in around 20\% of cases [45]. Recently Chao [46] showed the utility of PET/CT in assisting RT treatment planning of patients with potentially curable lymph node metastases. This new information together with the new radiotherapy tools (such as intensity-modulated radiation therapy) allow a decrease in the dose to surrounding healthy tissues, as well as an increase to the target [41,47-50].

\subsection{Re-Staging}

An early detection and an accurate staging of disease are crucial elements in order to plan the therapeutic strategy and to improve prognosis [51]. In asymptomatic patients previously treated for cervical cancer, the increase of serum levels of markers such as CEA, Ca19.9 and Ca125 is one of the signs of disease recurrence. However, tumour markers are non disease specific and do not give indications about the site of relapse and the amount of disease. In disease re-staging, conventional CECT and MRI are the most frequently used imaging modalities, even if some limitations should be taken into account: first of all, they are generally limited to one body district (pelvis and/or abdomen); secondly, they are often unable to identify cancer relapse in body districts which present post-surgical or post-radiotherapy scars (good sensitivity, but low specificity levels); thirdly, they are inaccurate in characterizing small lymph nodes and in detecting the peritoneal disease [52]. Therefore, as described by van der Weldt [53] the results of the above-mentioned exams are often inconclusive and equivocal, thus justifying the use of PET. In this study the ${ }^{18}$ F-FDG-PET/CT scan showed a good sensitivity and specificity: $92 \%$ and $93 \%$ respectively. Furthermore, as reported in Table 1, different authors in the recent years investigated the usefulness of PET(/CT) in the suspect of recurrence, and all of them showed high sensitivity and specificity levels. In fact, PET gives a metabolic characterization of the body structures independent to their anatomy, also allowing the investigation of critical regions in which anatomy has been modified [54]. Given this and due to the possibility of identifying distant metastasis, the therapeutic strategies are changed in about $25 \%$ of patients after PET exam [26,28].

Table 1. Sensitivity and specificity levels (in percentages) of ${ }^{18} \mathrm{~F}$-FDG-PET(/CT) in the assessment of disease recurrence in cervical cancer.

\begin{tabular}{lccc}
\hline Authors, year Ref & No. of patients & Sensitivity (\%) & Specificity (\%) \\
\hline Sakurai, 2005 [26] & 25 & 91.5 & 57.1 \\
Lin, 2006 [24] & 260 & 77.3 & 96.3 \\
Amit, 2006 [24] & 28 & 60 & 94 \\
Sironi, 2007 [22] & 25 & 93 & 100 \\
Chung, 2007 [12] & 52 & 90.3 & 81 \\
Husain, 2007 [25] & 20 & 100 & 73 \\
Kitajima, 2008 [17] & 30 & 93 & 93 \\
van der Weldt [53] & 40 & 92 & 93 \\
\hline
\end{tabular}


As regards the follow-up, Brooks [4] demonstrated that PET/CT presents a good accuracy in detecting recurrences in both asymptomatic and symptomatic women. In his study, it was interestingly showed that the survival of women with asymptomatic recurrences was superior to the symptomatic subjects.

As regards the prognosis, the modifications of the ${ }^{18}$ F-FDG-uptake during the course of the treatment represents an important predictor of tumour response and patient's survival [55]. Nishiyama [19] evaluated the role of PET in monitoring the neo-adjuvant therapy in patients with advanced stages of gynaecological cancers. In his study, PET showed an accuracy of $85 \%$ in predicting response to the treatment, when the SUVmax was decreased more than $65 \%$. However, larger studies are needed to better define the PET role in this context [56].

\section{ENDOMETRIAL CANCER}

Endometrial cancer is a common malignant disease, being the fourth cancer in post-menopausal women. In Europe, about 1 out of 20 new cancers' cases interests the endometrium and in the United States the incidence rate is about 40,000 new cases/year [57,58]. As in cervical cancer, the surgical-pathological FIGO staging system is used to address patients to the more appropriate treatment. Patients with FIGO stage IA and IB (involvement of less than $50 \%$ of myometrium thickness) can be treated with a tumour resection, while patients with stage IC (involvement of more than $50 \%$ of myometrium thickness) the para-aortic lymphnode dissection and the use of adjuvant chemo-radiotherapy is one of the proposed therapeutic strategy, due to the fact that this stage has a greater incidence of nodal and distant metastasis, with a worse prognosis [59]. Generally, patients with the clinical suspect of deep myometrial invasion undergo further examinations (CT or MRI), in order to assess the extra uterine disease spread.

The role of ${ }^{18} \mathrm{~F}$-FDG-PET/CT in the management of this neoplasm is not well defined due to the lack of consistent data in the literature, and due to this reason uterine corpus cancer is not included among appropriate applications in oncology [45].

Few small studies assessed the validity of PET alone in the evaluation of primary endometrial cancer, showing good levels of sensitivity (range 83.3-96.7\% [59-61]). Torizuka et al. affirmed the feasibility of PET study in the assessment of myometrial involvement, reporting a better diagnostic accuracy than MRI (86.4\% versus $77.3 \%$ respectively) [59]. In fact, in this study the SUV of the tumour correlated significantly with the depth of the tumour invasion. Furthermore, the authors used SUV of the primary tumour, dichotomized to 12 , in order to predict the depth of the tumour invasion. They showed that SUV $<12$ correlated significantly with superficial invasion, while SUV $>12$ correlated with profound invasion of the tumour. However, due to the limited spatial resolution of the PET study, a limited size of invasion could be missed, leading to false negative PET results [62]. Recently, two randomized trials showed that in early stages of endometrial cancer the routine pelvic lymphadenectomy improves the staging, furthermore this surgical staging correlated with prognosis of the patients. However, the advantage gained was only further knowledge, since no survival benefits were observed in these patients. On the contrary, women that undergo surgical staging have increase the risks of complications. Therefore, the goal of the non-invasive staging in these women would be to select those patients in whom the surgical staging could improve prognosis not only the staging [63-65]. In regard to PET/CT, Park et al. showed in a population of 53 patients weak levels of sensitivity and specificity in the staging of primary lesions (89.4 and 50.5\%, respectively) and in the staging of regional lymph nodes (69.2 and $90.3 \%$, respectively). On the other hand, high accuracy levels were reported in the detection of distant metastasis. The authors concluded that there are two main advantages of ${ }^{18} \mathrm{~F}$-FDG$\mathrm{PET} / \mathrm{CT}$ in the preoperative assessment of endometrial cancer: the good negative predictive value in predicting lymph node metastasis, that allows avoidance of surgical staging in poor candidates for such procedure; the high accuracy in detecting distant metastasis [66]. However, again it must be reminded that in the above mentioned study, the negative predictive value (NPV) was good but not excellent (98.9\% in the pelvic evaluation, decrease in $87.5 \%$ for the para-aortic nodes). Furthermore the NPV was further investigated by Signorelli [63]. It was indicated that in high risk early stages the high NPV could be useful to avoid systemic lymphaedenectomy. In such cases a de-bulking surgery of the involved nodes could be sufficient. With the decrease of lymph node dimension, a progressive significant reduction of sensitivity has been observed by Kitajima et al.: $93.3 \%$ for lesions greater than $10 \mathrm{~mm}, 66.7 \%$ for lesions between 5 and 9 $\mathrm{mm}$, and $16.7 \%$ for lesions smaller than $4 \mathrm{~mm}$ [67] Furthermore, Inubashiri [68] observed in recent work that ${ }^{18}$ F-FDG-PET/CT cannot change the medical management of the patients if a MRI is previously performed.

As indicated by some authors, in endometrial cancer the major contribution of ${ }^{18} \mathrm{~F}$-FDG-PET/CT could be in the early assessment of disease recurrence after therapy $[60,69]$. The good performance of ${ }^{18} \mathrm{~F}$-FDG-PET/CT has been confirmed by Kitajima et al. in a recent paper including 30 patients, that showed an overall patient-based sensitivity, specificity and accuracy of 93\% [70]. 
In conclusion, in endometrial cancer, the most relevant indications obtained by PET are: the possibility of detecting disease recurrence in asymptomatic patients presenting an increase of the Ca125 serum levels; the ability in distinguishing fibrotic tissue from viable lesions after the treatment; the metabolic definition of the radiotherapy treatment planning. Encouraging results are also available in the assessment of lymph nodes and distant metastasis during the disease staging.

\section{UTERINE SARCOMAS}

Uterine sarcomas are rare neoplastic diseases which represent about a 2-4\% of all uterine malignancies [71]. The histology is heterogeneous, but the more represented variety are the leiomyosarcoma, the carcinosarcoma and the endometrial sarcoma. These malignancies present an extremely poor prognosis and, despite their rarity, they are responsible for a large number of deaths every year, in women with uterine cancer. As with the benign uterine leiomyoma, clinical features are represented by vaginal bleeding and pain. Therefore, during the diagnostic process, the differential diagnosis is crucial. Unfortunately, in the majority of cases this is obtained only after surgery at the histopathological examination, because morphological imaging (US and MRI) is often inconclusive.

Therapeutic options in these cancers actually include the surgery (hysterectomy) when the cancer is localized, and chemotherapy if the tumour is locally and/or distantly extended. During the follow-up about one half of Stage I cases develop a recurrence [72]. In the management of sarcomas (soft tissues and bone sarcoma), the current literature evidences discordant data on the accuracy and the usefulness of ${ }^{18} \mathrm{~F}$-FDG-PET scan due to the small number and to the heterogeneity of the included cases; however, PET seems to be capable of differentiating between low and high grade sarcomas, and in the evaluation of residual disease after therapy [73]. In the case of uterine sarcomas, some authors suggest the use of PET study to distinguish between leiomyomas and leiomyosarcomas [74]. The identification of distant metastases could be another reason for the execution of a PET scan in the staging of these patients. However, a recent study presented that only $9 \%$ of patients performed an ${ }^{18} \mathrm{~F}$-FDG-PET scan as part of perioperative imaging. [75].

Despite the spare chemotherapy response the precocious and accurate detection of disease recurrence is crucial. However, an effective diagnostic strategy for an early identification of relapse is missing, and specific serological tumour markers are not available. Even if, at this moment, there is not enough evidence to validate the use of this exam in the follow up of asymptomatic patients and in the evaluation of clinically suspected recurrences, in this scenario ${ }^{18} \mathrm{~F}$-FDG-PET/CT could be useful. In fact in a recent study Ho and colleagues [76] showed that PET scan could change the management in falsely inoperable patients or showing distant metastases. On the other hand Park et al. [77] showed that the PET scan has a good impact in the surveillance of patients with uterine sarcomas. In fact, in this study, PET scan, show a very good accuracy $(94,4 \%)$ in women with suspected recurrence and a good sensitivity $(87,5 \%)$ in asymptomatic women and contribute to change the treatment in $1 / 3$ of the population. The results of the few small reports are encouraging in this way, but not completely in agreement [72,76,77].

Further, clinical studies are needed to define the role of ${ }^{18} \mathrm{~F}$-FDG-PET/CT in uterine sarcomas.

\section{CONCLUSIONS}

In this review, we evaluated the role of ${ }^{18} \mathrm{~F}$-FDG-PET/ CT in uterine malignancies. This safe, non invasive, imaging modality gives useful metabolic and anatomic information, and, as in the other field of oncology, it is beginning to play an important role in the management of these patients. Despite the relatively low number of studies, its usefulness in the assessment of lymph-nodal involvement at the staging, in the detection and staging of disease recurrence, and in the evaluation of the response after chemo- and radio-therapy has been proved. In fact the use of PET/CT in uterine cancer seems to be controversial. Despite numerous and rigorous study demonstrated the utility, gynecologic oncologist are not very enthusiastic of this exam. In fact, such as Kizer et al. [78] demonstrated in a recent study, when $83 \%$ of them routinely order CT scan, only $28 \%$ of them routinely order PET/CT scan. Some of them believe that PET/CT does not provide useful prognostic information whereas others causes could be the difficulty to obtain third party payment from the private paying clients. It seems that the better staging of metastatic lymph nodes or distant metastases, the very good negative predictive value in early stages, the correlation of before/after treatment PET result with the overall survival, data already available, have little impact and do not convince the gynecologic oncologist. However, these data are good sources for the planning of other prospective studies that could have a greater impact.

\section{REFERENCES}

[1] Tatsumi M, Cohade C, Bristow R.E. and Wahl R.L. (2009) Imaging uterine cervical cancer with FDG-PET/ 
CT: Direct comparison with PET. Molecular Imaging and Biology, 11(4), 229-235.

[2] National Cancer Institute, Cervical Cancer, Bethesda (MD). www.cancer.gov/cancertopics/types /cervical

[3] Arbyn, M., Raifu, A.O. and Ferlay, J. (2007) Burden of cervical cancer in Europe. Annals of Oncology, 18(10), 1708-1715.

[4] Brooks, R.A, Rader, J.S., Dehdashti, F., Mutch, D.G., Powell, M.A., Thaker, P.H., Siegel, B.A. and Grigsby, P.W. (2009) Surveillance FDG-PET detection of asymptomatic recurrences in patients with cervical cancer. $G y$ necologic Oncolology, 112(1), 104-109.

[5] Jemal, A., Siegel, R., Ward, E., Hao, Y., Xu, J. and Thun, M.J. (2009) Cancer statistics. CA: A Cancer Journal for Clinicians, 59(4), 225-249.

[6] Jemal, A., Siegel, R., Ward, E., Hao, Y., Xu, J., Murray, T. and Thun, M.J. (2008) Cancer statistics, 2008. CA: A Cancer Journal for Clinicians, 58(2), 71-96.

[7] Loft, A., Berthelsen, A.K., Roed, H., Ottosen, C., Lundwall, L., Knudsen, J., Nedergaard, L., Højgaard, L. and Engelholm, S.A. (2007) The diagnostic value of PET/CT scanning in patients with cervical cancer: A prospective study. Gynecologic Oncolology, 106(1), 29-34.

[8] Pecorelli, S., Benedet, J.L., Creasman, W.T. and Shepherd J.H. (1999) FIGO staging of gynecologic cancer. 1994-1997 FIGO committee on gynecologic oncology. international federation of gynecology and obstetrics. International Journal of Gynaecology and Obstetrics, 65(3), 243-269.

[9] Park, W., Park, Y.J., Huh. S.J., Kim, B.G., Bae, D.S., Lee J., Kim, B.H., Choi, J.Y., Ahn, Y.C. and Lim, D.H. (2005) The usefulness of MRI and PET imaging for the detection of parametrial involvement and lymph node metastasis in patients with cervical cancer. Japanese Journal of Clinical Oncology, 35(5), 260-264.

[10] Grigsby, P.W. and Herzog, T.J. (2001) Current management of patients with invasive cervical carcinoma. Clinical Obstetrics and Gynecology, 44(3), 531-537.

[11] Nicolet, V., Carignan, L., Bourdon, F. and Prosmanne, O. (2000) MR imaging of cervical carcinoma: A practical staging approach. Radiographics, 20(6), 1539-1549.

[12] Kumar, R. and Dadparvar, S. (2007) 18F-Fluoro-2-deoxy-D-glucose-Positron Emission Tomography (PET)/ PET-Computed Tomography in carcinoma of the cervix. Cancer, 110(8), 1650-1653.

[13] Chung, H.H., Kim, S.K., Kim, T.H., Lee, S., Kang, K.W., Kim, J.Y. and Park, S.Y. (2006) Clinical impact of 18F-FDG-PET imaging in post-therapy surveillance of uterine cervical cancer: From diagnosis to prognosis. Gynecologic Oncolology, 103(1), 165-170.

[14] Ryu, S.Y., Kim, M.H., Choi, S.C., Choi, C.W. and Lee, K.H. (2003) Detection of early recurrence with 18F-18FFDG PET in patients with cervical cancer. Journal of Nuclear Medicine, 44(3), 347-352.

[15] Harry, V.N. (2010) Novel imaging techniques as response biomarkers in cervical cancer. Gynecologic Oncolology, 116(2), 253-261.

[16] Lerman, H., Metser, U., Grisaru, D., Fishman, A., Lievshitz, G. and Even-Sapir, E. (2004) Normal and abnormal 18F-18F-FDG endometrial and ovarian uptake in pre- and post- menopausal patients: Assessment by PET/ CT. Journal of Nuclear Medicine, 45(2), 266-271.
[17] Kidd, E.A., Siegel, B.A., Dejdashti, F. and Grigsby, P.W. (2007) The standardized uptake value for F18-Fluorodeoxyglucose is a sensitive predictive biomarker for cervical cancer treatment response and survival. Cancer, 110(8), 1738-1744.

[18] Xue, F., Lin, L.L., Dehdashti, F., Miller, T.R., Siegel, B.A. and Grigsby, P.W. (2006) F18 fluorodeoxyglucose uptake in primary cervical cancer as an indicator of prognosis after radiation therapy. Gynecologic Oncolology, 101(1), 146-151.

[19] Nishiyama, Y., Yamamoto, Y., Kanenishi, K., Ohno, M., Hata, T., Kushida, Y., Haba, R. and Ohkawa, M. (2008) Monitoring the neoadjuvant therapy response in gynecological cancer patients using FDG PET. European Journal of Nuclear Medicine and Molecular Imaging, 35(2), 287-295.

[20] Yoshida, Y., Kurokawa, T., Kawahara, K., Yagihara, A., Tsuchida, T., Okazawa, H., Fujibayashi, Y., Yonekura, Y. and Kotsuji, F. (2004) Metabolic monitoring of advanced uterine cervical cancer neoadjuvant chemotherapy by using [F-18]-Fluorodeoxyglucose positron emission tomography: Preliminary results in three patients. Gynecologic Oncolology, 95(3), 597-602.

[21] Kidd, E.A., Spencer, C.R., Huettner, P.C., Siegel, B.A., Dehdashti, F., Rader, J.S. and Grigsby, P.W. (2009) Cervical cancer histology and tumor differentiation affect 18F-fluorodeoxyglucose uptake. Cancer, 115(15), 35483554.

[22] Lee, Y.Y., Choi, C.H., Kim, C.J., Kang, H., Kim, T.J., Lee, J.W., Lee, J.H., Bae, D.S. and Kim, B.G. (2009) The prognostic significance of the SUVmax (maximum standardized uptake value for F-18 fluorodeoxyglucose) of the cervical tumor in PET imaging for early cervical cancer: Preliminary results. Gynecologic Oncolology, 115(1), 65-68.

[23] Kim, S.K., Choi, H.J., Park, S.Y., Lee, H.Y., Seo, S.S., Yoo, C.W., Jung, D.C., Kang, S. and Cho, K.S. (2009) Additional value of MR/PET fusion compared with PET/CT in the detection of lymph node metastases in cervical cancer patients. European Journal of Cancer, 45(12), 2103-2109.

[24] Selman, T.J., Mann, C., Zamora, J., Appleyard, T.L. and Khan, K. (2008) Diagnostic accuracy of tests for lymph node status in primary cervical cancer: A systematic review and a metanalysis. Canadian Medical Association Journal, 178(7), 855-862.

[25] Yildirim, Y., Sehirali, S., Avci, M.E., Yilmaz, C., Ertopcu K., Tinar, S., Duman, Y. and Sayhan, S. (2008) Integrated PET/CT for the evaluation of paraaortic nodal metastasis in locally advanced cervical cancer patients with negative conventional CT findings. Gynecologic Oncolology, 108(1), 154-159.

[26] Havrilesky, L.J., Kulasingam, S.L., Matchar, D.B. and Myers, E.R. (2005) 18F-FDG-PET for management of cervical and ovarian cancer. Gynecologic Oncolology, 97(1), 183-191.

[27] Husain, A., Akhurst, T., Larson, S., Alektiar, K., Barakat, R.R. and Chi, D.S. (2007) A prospective study of the accuracy of 18Fluorodeoxyglucose positron emission tomography (18F-FDG PET) in identifying sites of metastasis prior to pelvic exenteration. Gynecologic Oncolology, 106(1), 177-180. 
[28] Nakamoto, Y., Saga, T. and Fujii, S. (2005) Positron emission tomography application for gynecologic tumors. International Journal of Gynecological Cancer, 15(5), 701-709.

[29] Roh, J.W., Seo, S.S., Lee, S., Kang, K.W., Kim, S.K., Sim, J.S., Kim, J.Y., Hong, E.K., Cho, D.S., Lee, J.S. and Park, S.Y. (2005) Role of positron emission tomography in pretreatment lymph node staging of uterine cervical cancer: A prospective surgicopathologic correlation study. European Journal of Cancer, 41(14), 2086-2092.

[30] Unger, J.B., Lilien, D.L., Caldito, G., Ivy, J.J., Charrier, A. and Bellaire, B. (2007) The prognostic value of pretreatment 2-[18F]-fluoro-2-deoxy-D-glucose positron emission tomography scan in women with cervical cancer. International Journal of Gynecological Cancer, 17(5), 1062-1067.

[31] Wright, J.D., Dehdashti, F., Herzog, T.J., Mutch, D.G., Huettner, P.C., Rader, J.S., Gibb, R.K., Powell, M.A., Gao, F., Siegel, B.A. and Grigsby, P.W. (2005) Preoperative lymph node staging of early-stage cervical carcinoma by [18F]-fluoro-2-deoxy-D-glucose-positron emission tomography. Cancer, 104(11), 2484-2491.

[32] Rezvani, M. and Shaaban, A. (2009) Imaging of cervical pathology. Clinical Obstetrics and Gynecology, 52(1), 94-111.

[33] Sironi, S., Buda, A., Picchio, M., Perego, P., Moreni, R., Pellegrino, A., Colombo, M., Mangioni, C., Messa, C. and Fazio, F. (2006) Lymph node metastasis in patients with clinical early-stage cervical cancer: Detection with integrated 18F-FDG PET/CT. Radiology, 238(1), 272279.

[34] Choi, H.J., Roh, J.W., Seo, S.S., Lee, S., Kim, J.Y., Kim, S.K., Kang, K.W., Lee, J.S., Jeong, J.Y and Park, S.Y. (2006) Comparison of the accuracy of magnetic resonance imaging and positron emission tomography/computed tomography in the presurgical detection of lymph node metastases in patients with uterine cervical carcinoma: A prospective study. Cancer, 106(4), 914-922.

[35] Chao, A., Ho, K.C., Wang, C.C., Cheng, H.H., Lin, G., Yen, T.C. and Lai, C.H. (2008) Positron emission tomography in evaluating the feasibility of curative intent in cervical cancer patients with limited distant lymph node metastasis. Gynecologic Oncology, 110(2), 172-178.

[36] Yen, T.C., See, L.C., Lai, C.H., Tsai, C.S, Chao, A., Hsueh, S., Hong, J.H., Chang, T.C. and Ng, K.K. (2008) Standardized up-take value in paraaortic lymph nodes is a significant prognostic factor in patients with primary advanced squamous cervical cancer. European Journal of Nuclear Medicine and Molecular Imaging, 35(3), 493501.

[37] Amit, A., Beck, D., Lowenstein, L., Lavie, O., Bar shalom, R., Kedar, Z. and Israel, O. (2006) The role of hybrid PET/CT in the evaluation of patients with cervical cancer. Gynecologic Oncology, 100(1), 65-69.

[38] Chou, H.H., Chang, T.C., Yen, T.C., Ng, K.K., Hsueh, S., Ya Ma, S., Chang, C.J., Huang, H.J., Chao, A., Wu, T.I., Jung, S.M., Lin, C.T., Huang, K.G. and Lai, C.H. (2006) Low value of [18F]-Fluoro-2-Deoxy-D-Glucose Positron Emission Tomography in primary staging of early stage cervical cancer before radical hysterectomy. Journal of Clinical Oncology, 24(1), 123-128.

[39] Boughanim, M., Leboulleux, S., Rey, A., Pham, C.T.,
Zafrani, Y., Duvillard, P,, Lumbroso, J., Haie-Meder, C., Schlumberger, M. and Morice, P. (2008) Histologic results of paraaortic lymphadenectomy in patients treated for stage IB2/II cervical cancer with negative [18F] Fluorodeoxy-glucose positron emission tomography scans in the paraaortic area. Journal of Clinical Oncology, 26(15), 2558- 2561.

[40] Kitajima, K., Murakami, K., Yamasaki, E., Kaji, Y. and Sugimura, K. (2009) Accuracy of integrated FDG-PET/ contrast-enhanced CT in detecting pelvic and paraaortic lymph node metastasis in patients with uterine cancer. European Radiology, 19(6), 1529-1536.

[41] Grosu, A.L., Piert, M., Weber, W., Jeremic, B., Piccio, M., Schratzenstaller, U., Zimmermann, F.B., Schwaiger, M. and Molls, M. (2005) Positron Emission Tomography for radiation treatment planning. Strahlentherapie und Onkologie, 181(8), 483-499.

[42] Gold, M.A., Tian, C., Whitney, C.W., Rose, P.G. and Lanciano, R. (2008) Surgical versus radiographic determination of paraaortic lymph node metastases before chemoradiation for locally advanced cervical carcinoma: A Gynecologic Oncology Group Study. Cancer, 112(9), 1954-1963.

[43] Dolezelova, H., Slampa, P., Ondrova, B., Gombosova, J., Sovadinova, S., Novotny, T., Bolcak, K., Ruzickova, J., Hynkova, L. and Forbelska, M. (2008) The impact of PET with 18FDG in radiotherapy treatment planning and in the prediction in patients with cervix carcinoma: Results of pilot study. Neoplasma, 55(5), 437-441.

[44] Boughanim, M., Leboulleux, S., Rey, A., Pham, C.T., Zafrani, Y., Duvillard, P., Lumbroso, J., Haie-Meder, C., Schlumberger, M. and Morice, P. (2008) Histologic results of paraaortic lymphadenectomy in patients treated for stage IB2/II cervical cancer with negative [18F] fluorodeoxyglucose positron emission tomography scans in the paraaortic area. Journal of Clinical Oncology, 26(15), 2558-2561.

[45] Regione Emilia Romagna. ${ }^{18}$ F-FDG-PET in oncologia. Criteri per un uso appropriato, 2006.

[46] Chao, A., Ho, K.C., Wang, C.C., Cheng, H.H., Lin, G., Yen, T.C. and Lai, C.H. (2008) Positron emission tomography in evaluating the feasibility of curative intent in cervical cancer patients with limited distant lymph node metastases. Gynecologic Oncology, 110(2), 172-178.

[47] Lin, C.T., Yen, T.C., Chang, T.C., Ng, K.K., Tsai, C.S., Ho, K.C. and Lai, C.H. (2006) Role of [18F] fluoro-2deoxy-D-glucose positron emission tomography in re-recurrent cervical cancer. International Journal of Gynecological Cancer, 16(6), 1994-2003.

[48] Esthappan, J., Mutic, S., Malyapa, R.S., Grigsby, P.W., Zoberi, I., Dehdashti, F., Miller, T.R., Bosch, W.R. and Low, D.A. (2004) Treatment planning guidelines regarding the use of CT/PET-guided IMRT for cervical carcinoma with positive paraaortic lymph nodes. International Journal of Radiation Oncology, Biology, Physics, 58(4), 1289-1297.

[49] Esthappan, J., Chaudhari, S., Santanam, L., Mutic, S., Olsen, J., MacDonald, D.M., Low, D.A., Singh, A.K. and Grigsby, P.W. (2008) Prospective clinical trial of Positron Emission Tomography/Computed Tomography imageguided intensity-modulated radiation therapy for cervical carcinoma with positive paraaortic lymph nodes. Inter- 
national Journal of Radiation Oncology, Biology, Physics, 72(4), 1134-1139

[50] Lin, L.L., Mutic, S., low, D., LaForest, R., Vicic, M., Zoberi, I., Miller, T.R. and Grigsby, P.W. (2007) Adaptive brachytherapy treatment planning for cervical cancer using 18F-FDG-PET. Journal of Radiation Oncology, Biology, Physics, 67(1), 91-96.

[51] Kitajima, K., Murakami, K., Yamasaki, E., Hagiwara, S., Fukasawa, I., Inaba, N., Kaji, Y. and Sugimura, K. (2008) Performance of 18F-FDG-PET/CT in the diagnosis of recurrent endometrial cancer. Annals of Nuclear Medicine, 22(2), 103-109.

[52] Grisaru, D., Almog, B., Levine, C., Metser, U., Fishman A., Lerman, H., Lessing, J.B. and Even-Sapir, E. (2004) The diagnostic accuracy of 18F-fluorodeoxyglucose PET/ $\mathrm{CT}$ in patients with gynecological malignancies. Gynecologic Oncoloy, 94(3), 680-684.

[53] van der Veldt, A.A., Buist, M.R., van Baal, M.W., Comans, E.F., Hoekstra, O.S. and Molthoff, C.F. (2008) Clarifying the diagnosis of clinically suspected recurrence of cervical cancer: Impact of 18F-FDG PET. Journal of Nuclear Medicine, 49(12), 1936-1943.

[54] Bjurberg, M., Kjellen, E., Ohlsson, T., Ridderheim, M. and Brun, E. (2007) 18F-FDG-PET in cervical cancer: staging, re-stagting and follow up. Acta Obstetricia et Gynecologica, 86(11), 1385-1391.

[55] Schwarz, J.K., Siegel, B.A., Dehdashti, F. and Grigsby, P.W. (2007) Association of posttherapy positron emission tomogramphy with tumor response and survival in cervical carcinoma. Journal of the American Medical Association, 289(19), 2289-2295.

[56] Schwarz, J.K., Grigsby, P.W., Dehdashti, F. and Delbeke, D. (2009) The role of 18F-FDG PET in assessing therapy response in cancer of the cervix and ovaries. Journal of Nuclear Medicine, 50 (Suppl 1), 64S-73S.

[57] Bray, F., Dos, S.S.I., Moller, H. and Weiderpass, E. (2005) Endometrial cancer incidence trends in Europe: Underlying determinants and prospects for prevention. Cancer Epidemiology, Biomarkers \& Prevention, 14(5), 11321142.

[58] Jemal, A., Tiwari, R.C., Murray, T., Ghafoor, A., Samuel, A., Ward, E., Feuer, E.J. and Thun, M.J. (2004) American Cancer Society. Cancer Statistics, 2004. CA: A Cancer Journal for Clinicians, 54(1), 8-29.

[59] Torizuka, T., Nakamura, F., Takekuma, M., Toshihiko, K., Ogusu, T., Yoshikawa, E., Okada, H., Maeda, M. and Ouchi, Y. (2006) 18F-FDG PET for the assessment of myometrial infiltration in clinical stage I uterine corpus cancer. Nuclear Medicine Communications, 27(6), 481487.

[60] Chao, A., Chang, T.C., Ng, K.K., Hsueh, S., Huang, H.J., Chou, H.H., Tsai, C.S., Yen, T.C., Wu, T.I. and Lai, C.H. (2006) 18F-FDG PET in the management of endometrial cancer. European Journal of Nuclear Medicine and Molecular Imaging, 33(1), 36-44.

[61] Suzuki, R., Miyagi, E., Takahashi, N., Sukegawa, A., Suzuki, A., Koike, I., Sugiura, K., Okamoto, N., Inoue, T. and Hirahara, F. (2007) Validity of positron emission tomography using fluoro-2-deoxyglucose for the preoperative evaluation of endometrial cancer. International Journal of Gynecological Cancer, 17(1), 890-896.

[62] Horowitz, N.S., Dehdashti, F., Herzog, T.J., Rader, J.S.,
Powell, M.A., Gibb, R.K., Grigsby, P.W., Siegel, B.A, Mutch, D.G. (2004) Prospective evaluation of 18F-FDGPET for detecting pelvic and para-aortic lymphnode metastasis in uterine corpus cancer. Gynecologic Oncology, 95(3), 546-551.

[63] Signorelli, M., Guerra, L., Buda, A., Picchio, M., Mangili G., Dell'Anna, T., Sironi, S. and Messa, C. (2009) Role of the integrated FDG PET/CT in the surgical management of patients with high risk clinical early stage endometrial cancer: Detection of pelvic nodal metastases. Gynecologic Oncology, 115(2), 231-235.

[64] ASTEC study group, Kitchener, H., Swart, A.M., Qian, Q., Amos, C. and Parmar, M.K. (2009) Efficacy of systematic pelvic lymphadenectomy in endometrial cancer (MRC ASTEC trial): A randomised study. Lancet, 373(9658), 125-136.

[65] Benedetti, P. P., Basile, S., Maneschi, F., Alberto, L. A., Signorelli, M., Scambia, G., Angioli, R., Tateo, S., Mangili, G., Katsaros, D., Garozzo, G., Campagnutta, E., Donadello, N., Greggi, S., Melpignano, M., Raspagliesi, F., Ragni, N., Cormio, G., Grassi, R., Franchi, M., Giannarelli, D., Fossati, R., Torri, V., Amoroso, M., Crocè, C. and Mangioni, C. (2008) Systematic pelvic lympha-denectomy vs. no lymphad-enectomy in early-stage endometrial carcinoma: Randomized clinical trial. Journal of the National Cancer Institute, 100(23), 1707- 1716.

[66] Park, J.Y., Kim, E.N., Suh, D.S., Kim, J.H., Kim, Y.M., Kim, Y.T. and Nam, J.H. (2008) Comparison of the validity of magnetic resonance imaging and positron emission tomography/ computed tomography in the preoperative evaluation of patients with uterine corpus cancer. Gynecologic Oncology, 108(3), 486-492.

[67] Kitajima, K., Murakami, K., Yamasaki, E., Fukasawa, I., Inaba, N., Kaji, Y. and Sugimura, K. (2008) Accuracy of 18F-FDG PET/CT in detecting pelvic and paraaortic lymph node metastasis in patients with endometrial cancer. American Journal of Roentgenology, 190(6), 16521658.

[68] Inubashiri, E., Hata, K., Kanenishi, K., Shiota, A., Ohno, M., Yamamoto, Y., Nishiyama, Y., Ohkawa, M. and Hata, T. (2009) Positron emission tomography with the glucose analog [F]-fluoro-2-deoxy-D-glucose for evaluating pelvic lymph node metastasis in uterine corpus cancer: Comparison with CT and MRI findings. The Journal of $\mathrm{Ob}$ stetrics and Gynaecology Research, 35(1), 26-34.

[69] Sironi, S., Picchio, M., Landoni, C., Galimberti, S., Signorelli, M., Bettinardi, V., Perego, P., Mangioni, C., Messa, C. and Fazio, F. (2007) Post-therapy surveillance of patients with uterine cancers: Value of integrated 18F-FDG-PET/CT in the detection of recurrence. European Journal of Nuclear Medicine and Molecular Imaging, 34(4), 472-479.

[70] Kitajima, K., Murakami, K., Yamasaki, E., Hagiwara, S., Fukasawa, I., Inaba, N., Kaji, Y. and Sugimura, K. (2008) Performance of 18F-FDG-PET/CT in the diagnosis of recurrent endometrial cancer. Annals of Nuclear Medicine, 22(2), 103-109.

[71] Ho, K.C., Lai, C.H., Wu, T.I., Ng, K.K., Yen, T.C., Lin, G., Chang, T.C., Wang, C.C., Hsueh, S. and Huang, H.J. (2008) 18F-fluoro-deoxyglucose positron emission tomography in uterine carcinosarcoma. European Journal of Nuclear Medicine and Molecular Imaging, 35(3), 
484-492.

[72] Murakami, M., Tsukada, H., Shida, M., Watanabe, M., Maeda, H., Koido, S., Hirasawa, T., Muramatsu, T., Miyamoto, T., Nasu, S., Yasuda, S., Kajiwara, H. and Yasuda, Ide, M. (2006) Whole-body positron emission tomography with F18 fluorodeoxyglucose for the detection of recurrence in uterine sarcomas. International journal of gynecological Cancer, 16(2), 854-860.

[73] Bastiaannet, E., Groen, H., Jager, P.L., Cobben, D.C., van der Graaf, W.T., Vaalburg, W. and Hoekstra, H.J. (2004) The value of 18F-FDG-PET in the detection, grading and response to therapy of soft tissue and bone sarcomas; a systematic review and meta-analysis. Cancer Treatment Reviews, 30(1), 83-101.

[74] Rebollo, A.A.C., Ramos, F.C., Bellon G.M.E., Cabello G. D., Gallego P.M, Rodriguez, F.A. and Llamas E.J.M. (2007) Positron Emission Tomography with 18F-fluorodeoxyglucose in patients with uterine sarcoma. Revista Española de Medicina Nuclear, 26(4), 189-195.

[75] Nugent, E.K., Zighelboim, I., Case, A.S., Gao, F., Thaker,
P.H., Rader, J.S., Mutch, D.G. and Massad, L.S. (2009) The value of perioperative imaging in patients with uterine sarcomas. Gynecologic Oncology, 115(1), 37-40.

[76] Ho, K.C., Lai, C.H., Wu, T.I., Ng. K.K., Yen. T.C., Lin, G., Chang, T.C., Wang. C.C., Hsueh, S. and Huang, H.J. (2008) 18F-fluorodeoxyglucose positron emission tomography in uterine carcinosarcoma. European Journal of Nuclear Medicine and Molecular Imaging, 35(3), 484492.

[77] Park, J.Y., Kim, E.N., Kim, D.Y., Suh, D.S., Kim, J.H., Kim, Y.M., Kim, Y.T. and Nam, J.H. (2008) Role of PET or PET/CT in the post-therapy surveillance of uterine sarcoma. Gynecologic Oncology, 109(2), 255-262.

[78] Kizer, N.T., Zighelboim, I., Case, A.S., Dewdney, S.B., Thaker, P.H. and Massad, L.S. (2009) The role of PET/CT in the management of patients with cervical cancer: Practice patterns of the members of the Society of Gynecologic Oncologists. Gynecologic Oncology, 114(2), 310-314.

\section{ABBREVIATIONS}

PET: Positron Emission Tomography;

CT: Computed Tomography;

$\mathrm{PET} / \mathrm{CT}$ : integrated PET/CT;

CECT: Contrast Enhancement Computed Tomography;

18F-FDG: 18F-Fluoro-Deoxy-Glucose;

Pap-test: Papanicolau screening test;

FIGO: Federation of Gynaecologists and Obstetrics;

MRI: Magnetic Resonance Imaging;
SUVmax: Standardized Uptake Value;

DFS: Disease Free Survival;

GOG: Gynaecological Oncology Group;

RT: Radiotherapy;

CEA: Carcinoembryonic Antigen;

CA 19.9: Carbohydrate Antigen 19.9;

CA 125: Carbohydrate Antigen 125;

NPV: Negative Predictive Value;

US: Ultrasonography 